University of Wollongong

Research Online

Australian Institute for Innovative Materials -

Papers

Australian Institute for Innovative Materials

2012

\title{
All-polymer battery system based on polypyrrole (PPy)/para (toluene sulfonic acid) (pTS) and polypyrrole (PPy)/indigo carmine (IC) free standing films
}

Irin Sultana

University Of Wollongong, is988@uow.edu.au

Md. Mokhlesur Rahman

University Of Wollongong

Jiazhao Wang

University of Wollongong, jiazhao@uow.edu.au

Caiyun Wang

University of Wollongong, caiyun@uow.edu.au

Gordon G. Wallace

University of Wollongong, gwallace@uow.edu.au

See next page for additional authors

Follow this and additional works at: https://ro.uow.edu.au/aiimpapers

Part of the Engineering Commons, and the Physical Sciences and Mathematics Commons

Research Online is the open access institutional repository for the University of Wollongong. For further information contact the UOW Library: research-pubs@uow.edu.au 


\title{
All-polymer battery system based on polypyrrole (PPy)/para (toluene sulfonic acid) (pTS) and polypyrrole (PPy)/indigo carmine (IC) free standing films
}

\begin{abstract}
In this study, we introduce a novel all-polymer battery system based on conducting polymer (polypyrrole, PPy) doped with dopants of para (toluene sulfonic acid) ( $p T S$ ) and indigo carmine (IC), respectively. The performance of the systems consisting of polypyrrole-para (toluene sulfonic acid) (PPy-pTS) as cathode and polypyrrole-indigo carmine (PPy-IC) as anode in conjunction with either a polymer based electrolyte or a commercial organic electrolyte of $1 \mathrm{M} \mathrm{LiPF}_{6}$ in a 50:50 (v/v) mixture of ethylene carbonate (EC) and dimethyl carbonate (DMC) was evaluated. In the system, all the free-standing PPy-pTS and PPy-IC films were directly used without needing any metal substrate support to hold the electro active material. Electrochemical measurements demonstrated that the PPy-pTS/PPy-IC (commercial electrolyte) system exhibited a reversible discharge capacity of $36 \mathrm{mAh} \mathrm{g}^{-1}$ at $0.05 \mathrm{~mA} \mathrm{~cm}^{-2}$ after 50 cycles, is $92 \%$ of the initial discharge capacity. In the case of PPy-pTS/PPy-IC (polymer electrolyte), the reversible discharge capacity after 50 cycles was $16 \mathrm{mAh} \mathrm{g}^{-1}, 76 \%$ of the intial discharge capacity. This work deals with the fabrication of a novel all polymer battery system, with significant advantages in terms of capacity and reasonable stability. This may lead to a future generation of all polymer batteries that are suitable for implanted medical devices used in biological and biomedical systems. (c) 2012 Elsevier Ltd. All rights reserved.
\end{abstract}

\section{Keywords}

battery, system, polypyrrole, ppy, para, toluene, sulfonic, all, acid, films, pts, indigo, carmine, ic, free, standing, polymer

Disciplines

Engineering | Physical Sciences and Mathematics

\section{Publication Details}

Sultana, I., Rahman, M., Wang, J., Wang, C., Wallace, G. G. \& Liu, H. (2012). All-polymer battery system based on polypyrrole (PPy)/para (toluene sulfonic acid) (pTS) and polypyrrole (PPy)/indigo carmine (IC) free standing films. Electrochimica Acta, 83 209-215.

\section{Authors}

Irin Sultana, Md. Mokhlesur Rahman, Jiazhao Wang, Caiyun Wang, Gordon G. Wallace, and Hua-Kun Liu 
All-polymer battery system based on polypyrrole (PPy)/para (toluene sulfonic acid) (pTS) and polypyrrole (PPy)/indigo carmine (IC) freestanding films

Irin Sultana ${ }^{\mathrm{a}^{*}}$, M. M. Rahman ${ }^{\text {a, Jiazhao Wang }}{ }^{\text {a }}$, Caiyun Wang ${ }^{\text {b }}$, G.G. Wallace $^{b}$, Hua-Kun Liu ${ }^{a}$

${ }^{\mathrm{a}}$ Institute for Superconducting and Electronic Materials and ARC Centre of Excellence for Electromaterials Science, University of Wollongong, NSW 2522, Australia

${ }^{\mathrm{b}}$ Intelligent Polymer Research Institute and ARC Centre of Excellence for Electromaterials Science, University of Wollongong, NSW 2522, Australia

${ }^{*}$ Corresponding author

Irin Sultana

E-mail: is988@uowmail.edu.au

Tel: +61 242981447

Fax: +61 242215731 


\section{Abstract}

In this present study, we introduce a novel all-polymer battery system based on conducting polymer (polypyrrole, PPy) doped with redox-active compounds of para (toluene sulfonic acid) (pTS) and indigo carmine (IC), respectively. The performance of the systems consisting of polypyrrole-para (toluene sulfonic acid) (PPy-pTS) as cathode and polypyrrole-indigo carmine (PPy-IC) as anode in conjunction with gel polymer electrolyte and commercial electrolyte of $1 \mathrm{M} \mathrm{LiPF}_{6}$ are evaluated. In the system, all the free-standing PPy-pTS and PPyIC films were directly used without need any metal substrate to act as an electrical conductor. Electrochemical measurements demonstrated that the PPy-pTS/PPy-IC (commercial electrolyte) system exhibited a discharge capacity of $36 \mathrm{mAh} \mathrm{g}^{-1}$ at $0.05 \mathrm{~mA} \mathrm{~cm}{ }^{-2}$ after 50 cycles, which is around $92 \%$ of the initial discharge capacity. In the case of PPy-pTS/PPy-IC (polymer electrolyte), the discharge capacity retention was $16 \mathrm{mAh} \mathrm{g}^{-1}$, which is also $76 \%$ of the intial discharge capacity. The capacity degradation rate was calculated only 0.16 and 0.48 \%/cycle for the PPy-pTS/PPy-IC (commercial electrolyte) and PPy-pTS/PPy-IC (polymer electrolyte) system, respectively, confirming stable cycling performance of the both systems. Obviously, this work deals with the fabrication of a novel all polymer battery system, with significant advantages in terms of environmental friendliness, high capacity, and good cycling stability, which can lead to a future generation of polymer batteries to satisfy new market demand in the fields of energy storage devices.

Keywords: All-polymer battery; gel polymer electrolyte; redox-active compounds; Freestanding films, PPy-pTS cathode; PPy-IC anode. 


\section{Introduction}

The rapid progresses in electronics and increasing public awareness of environmental issues have recently been putting more pressure on clean sustainable energy sources. Thus, achieving the goals of non- or less toxic and more environmentally friendly materials as electrodes for energy storage devices has become mandatory if clean renewable technologies are to be developed for the future [1-3]. In this respect, the concept of all-polymer based batteries and supercapacitors, which was introduced almost three decades ago [4], has recently gained a renewed interest mainly due to the possibilities of manufacturing flexible and safe energy storage devices. The research within this field is currently undergoing an exciting development as new polymers, composites and paper-based devices are being developed [5]. To build an efficient electrochemical device using conducting polymers, three types of systems are usually considered: [6, 7] (i) symmetric p-doped/undoped systems wherein both electrodes are made of identical materials; (ii) asymmetric p-doped/undoped systems wherein the cathode and anode are made of different materials; and (iii) symmetric p-doped/n-doped systems. The p-doped/undoped systems typically give a cell voltage of about $1 \mathrm{~V}$ and aqueous electrolytes can be used. For the asymmetric designs cell voltages of up to about $1.5 \mathrm{~V}$ are possible to reach [5]. Although most classes of conducting polymers can be switched between an electrically neutral state and an oxidized (p-doped) state, very few polymers can be electrochemically reduced (n-doped). The coupling of an anode and cathode based on the same polymer in the neutral and the p-doped states results in a very small cell potential, and consequently conducting polymers that can only be p-doped are limited for use as cathodes in battery systems. However, by incorporating a large polymeric anion into a polymer film during deposition, a pseudo n-dopable response can be achieved [810]. The low mobility of the trapped anions results in charge compensation for these 
materials by cation transport and causes a negative shift in the oxidation potential, thus increasing the voltage separation between the reduced form of the polymer/polyanion electrode and the oxidized form of the polymer [11].

The recent work on the development of conducting polymer-based energy storage devices has involved development of both symmetrical and asymmetrical batteries and supercapacitors. Although flexible systems have been presented, very few of these [12, 13] may, however, be classified as prototypes for flexible all-polymer systems as electrodeposition of the polymers on metallic electrodes or metallic current collectors generally has been employed. In the development of flexible polymer fiber, non-aqueous batteries it has been shown [14] that two PPy films polymerized on platinum wires and separated by a polymeric hollow membrane can yield capacities of about $10 \mathrm{mAh} \mathrm{g}^{-1}$ over 30 cycles. It is interesting to note that freestanding flexible and paper-like films of PPy (and PPy films containing $\mathrm{LiFePO}_{4}$ particles), can be prepared by electrochemical polymerization on stainless steel substrates $[15,16]$, while the authors envisioned these as cathodes combined with CNT anodes in soft flexible batteries. Efforts have been made to develop all-polymer based devices utilizing electropolymerized polypyrrole (PPy) containing two different redox active anions, indigo carmine and 3-ethylbenzothiazoline-6-sulfonate, as thin layer electrodes on gold/titanium coated PET substrates [17]. An energy density of $8 \mathrm{Wh} \mathrm{kg}^{-1}$ and a power density of up to 10 $\mathrm{kW} \mathrm{kg}^{-1}$ were reported. A $50 \%$ loss of capacity, ascribed to overoxidation of PPy was, however, seen after 60 cycles indicating that the stability of the described system needs to be improved significantly. Similar stability problems were noted when using different functionalized polyerthiophenes (poly (3'-styryl-4,4"'-didecyloxyterthiophene) and poly (4,4”-didecyloxyterthiophene)) as anodes versus PPy cathodes [12]. The polymers were in this case electropolymerized on conducting and flexible mesh substrates (stainless steel and 
$\mathrm{Ni} / \mathrm{Cu}$ coated polyester, respectively) and the losses of capacity during cycling were ascribed to mechanical degradation of the polymer. A more stable system has been obtained with a claimed all-polymer battery containing a functionalized PTP based anode, a PPy cathode electrode and carbon-fiber mats or Ni/Cu-coated nonwoven polyester current collectors [13]. The reported discharge capacity was $35.4 \mathrm{mAh} \mathrm{g}^{-1}$ and no loss of capacity was seen after 50 charge and discharge cycles employing a current of $0.05 \mathrm{~mA} \mathrm{~cm}{ }^{-2}$. These results are encouraging and represent significant progress toward a flexible polymer-based device.

Nevertheless, most of the reported systems used metal substrate to coat or deposit active materials on it. Obviously, this kind of electrode is not suitable for fully flexible or bendable batteries as active material layers will be cracked or peeled off from the substrate when they are bent frequently. To avoid these drawbacks, mechanically flexible, soft, and free-standing electrode materials are required, indeed. We describe herein a novel system consists of PPy doped with redox-active compounds of para (toluene sulfonic acid) (pTS) (Fig. 1a) as cathode and indigo carmine (IC) (Fig.1b) as anode, respectively. All the free-standing PPy-pTS and PPy-IC films were successfully cycled between 3.0-0.01 V without need any metal substrate to act as an electrical conductor. Furthermore, electrochemical measurements have demonstrated that the system works very well both in commercial and gel polymer electrolyte, with significant advantages in terms of environmental safety issues, high capacity, and stable cycling performance compared with those previously reported results. 
<smiles>Cc1ccc(S(=O)(=O)[O-])cc1</smiles>

(a)

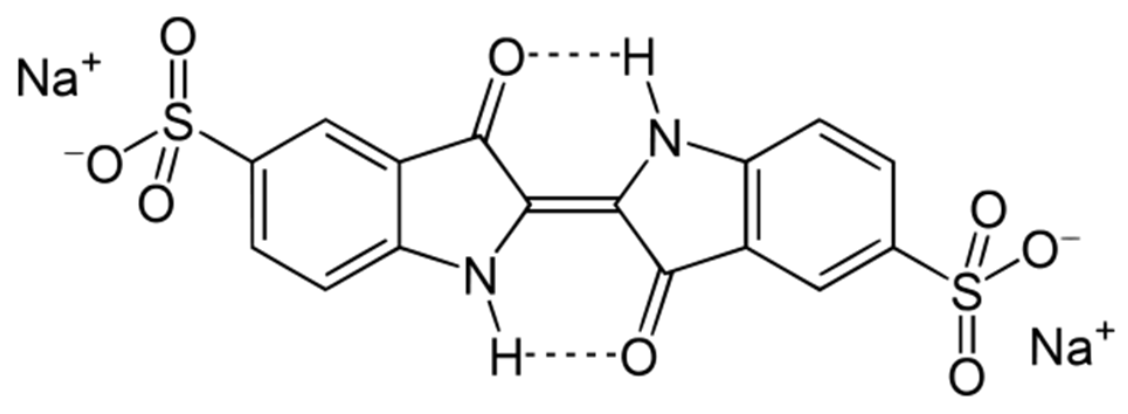

(b)

Fig.1. Molecular structure of a) para (toluene sulfonic acid) ( $p$ TS) and d) indigo carmine (IC).

\section{Experimental}

\subsection{Preparation of PPy-pTS free-standing cathode}

PPy-pTS films were electrodeposited using the galvanostatic technique at a current density of $0.2 \mathrm{~mA} \mathrm{~cm} \mathrm{~cm}^{-2}$ onto a polished stainless steel plate from a electrolyte solution prepared in deionized water with $0.1 \mathrm{~mol} \mathrm{l}^{-1}$ pyrrole (98\%, Sigma-Aldrich) and $0.1 \mathrm{~mol} \mathrm{l}^{-1}$ para (toluene sulfonic acid) sodium salt (95\%, Sigma-Aldrich). Pyrrole was first distilled and kept refrigerated until use. Dissolved oxygen was removed by bubbling the solution with nitrogen for at least 10 min prior to polymerization. A two electrodes electrochemical system was used for polymerization at room temperature $\left(27^{\circ} \mathrm{C}\right)$, where polished stainless steel plate (washed with acetone in an ultrasonic bath before use) used as a working electrode and stainless steel 
mesh as a counter electrode. After electropolymerization, the working electrode was rinsed with acetonitrile and was dried in oven, and then transferred into a solution of $0.75 \mathrm{M} \mathrm{LiClO}_{4}$ in dimethylformamide/acetonitrile (1:1 by volume). The PPy-pTS cathode films were converted into fully oxidized form by polarization at $+0.2 \mathrm{~V}$ (versus $\mathrm{Ag} / \mathrm{Ag}^{+}$in $0.01 \mathrm{M}$ $\mathrm{AgNO}_{3}, 0.1 \mathrm{M}$ tetrabutylammonium perchlorate (TBAP/CH $\left.\left.3 \mathrm{CN}\right)\right)$. The resultant films were peeled off from the polished stainless steel plate and were dried in a vacuum oven for 24 hours at room temperature. After drying, the prepared flexible free-standing films were cut into $1 \mathrm{~cm} \times 1 \mathrm{~cm}$ pieces and then transferred to an argon filled glove box.

\subsection{Preparation of PPy-IC free-standing anode}

Polypyrrole films doped with indigo carmine, PPy-IC, were also prepared using the same above mention method at a current density of $0.4 \mathrm{~mA} \mathrm{~cm}^{-2}$ onto a polished stainless steel plate in an aqueous $0.1 \mathrm{~mol} \mathrm{l}^{-1}$ solution of pyrrole (98\%, Sigma-Aldrich) saturated with $0.1 \mathrm{~mol} \mathrm{l}^{-1}$ indigo carmine solution (85\%, Sigma-Aldrich) under a $\mathrm{N}_{2}$ atmosphere. After electropolymerization, the working electrode was rinsed with dry acetonitrile and was dried in oven, and then transferred into a solution of $0.75 \mathrm{M} \quad \mathrm{LiClO}_{4}$ in dimethylformamide/acetonitrile (1:1 by volume). The PPy-IC anode films were converted into fully reduced form by polarization at $-1.3 \mathrm{~V}$ (versus $\mathrm{Ag} \mathrm{Ag}^{+}$in $0.01 \mathrm{M} \mathrm{AgNO}_{3}$, 0.1Mtetrabutylammonium perchlorate $\left(\mathrm{TBAP} / \mathrm{CH}_{3} \mathrm{CN}\right)$ ) reference electrode. The resultant films were then peeled off from the polished stainless steel plate, dried and cut to a small size of $1 \mathrm{~cm}^{2}$ to store in an argon-filled glove box. 


\subsection{Preparation of gel polymer electrolyte}

In a typical procedure, $1 \mathrm{~g}$ of PVDF-HFP was mixed with $50 \mathrm{ml}$ acetonitrile. The mixture was constant stirred and heated at $80{ }^{\circ} \mathrm{C}$ until dissolve the PVDF-HFP. The solution was cast onto a glass plate under ultra sonication for a period of $10 \mathrm{~min}$ and then, it was dried in room temperature for $24 \mathrm{hr}$ to get a film. The film was cut into required sizes (same as separator) and kept in glove box. The gel polymer electrolyte was obtained by soaking the films in $1 \mathrm{M}$ $\mathrm{LiPF}_{6}$ in a mixture of ethylene carbonate (EC) and dimethyl carbonate (DMC) (1:1 by volume, provided by MERCK, Germany) for $24 \mathrm{hr}$ before use it.

\subsection{Instrumental analysis}

Conductivity of the free-standing films was tested by Jandel multi-height four-point probes, connected to a Jandel resistivity test unit, model RM2, located in the Intelligent Polymer Research Institute (IPRI), University of Wollongong. A constant current of $1 \mathrm{nA}$ was applied to the outer two electrodes and the voltage difference between the two inner electrodes was measured. The morphologies of the films were obtained from field emission scanning electron microscopy (FE-SEM; JEOL JSM-7500FA equipped with energy dispersive X-ray spectroscopy (EDS) and an EDS mapping system). For infrared (IR) spectroscopy, all spectra were measured from 4000 to $500 \mathrm{~cm}^{-1}$, and the number of scans was typically 10, with a resolution of $2 \mathrm{~cm}^{-1}$.

\subsection{Electrochemical measurements}

CR 2032 coin-type cells were assembled in an Ar-filled glove box (Mbraun, Unilab, Germany) by stacking a gel polymer electrolyte between the PPy-IC free standing anode and 
PPy-pTS free standing cathode, as shown in Fig. 2. In the case of commercial electrolyte, a porous polypropylene separator containing liquid electrolyte was used between both electrodes. The electrolyte used was $1 \mathrm{M} \mathrm{LiPF}_{6}$ in a 50:50 (v/v) mixture of ethylene carbonate (EC) and dimethyl carbonate (DMC), provided by MERCK KgaA, Germany. Chargedischarge tests were carried out by using a battery testing device (Neware Electronic Co., China) interfaced to a computer with software. The system is capable of switching between charge and discharge automatically according to the pre-set cut-off potentials. The cells were cycled between 0.01-3.0 V with a constant current of $0.05 \mathrm{~mA} \mathrm{~cm}{ }^{2}$. Electrochemical impedance spectroscopy (EIS) was performed using a CHI 660C electrochemistry workstation. The AC amplitude was $5 \mathrm{mV}$, and the frequency range applied was $100 \mathrm{kHz}$ $0.01 \mathrm{~Hz}$.

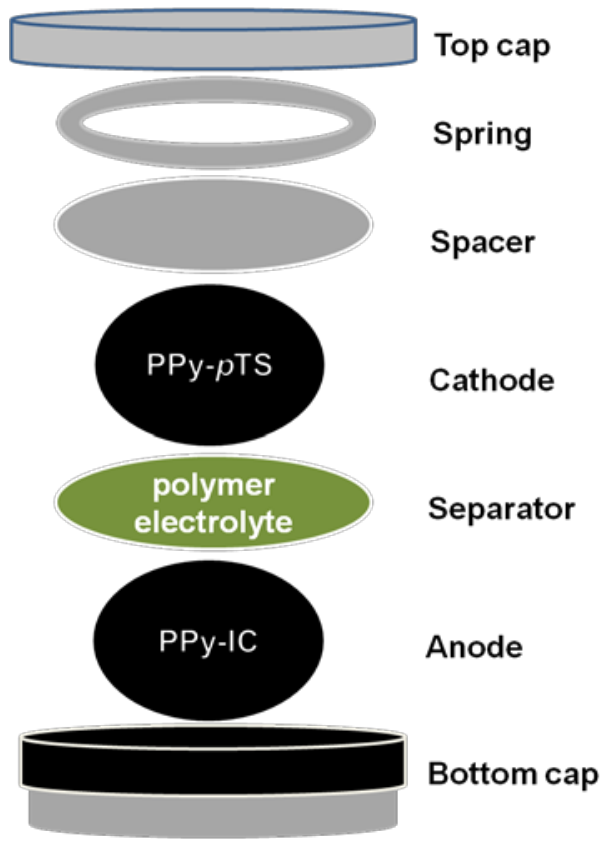

Fig. 2. Construction of CR 2032 coin type test cell. 


\section{Results and discussion}

Fig. 3 shows FESEM images of PPy-pTS (Fig.3a) and PPy-IC (Fig. 3b) electrodes illustrating the surface morphology of the free standing films. The morphology of the both films features a cauliflower-like nodule structure composed of microspherical grains, which is related to dopant intercalation in the polymeric chain [18]. The free-standing films can be rolled up (inset of Fig. 3(a) and Fig. 3 (b)), twisted, or bent to any curvature, and then returned to its original shape, while still maintaining its useful properties. Conductivity of the free-standing films was measured and it was 41.0 Scm ${ }^{-2}$ for the PPy-pTS with a thickness of $7.0 \mu \mathrm{m}$ and $7.9 \times 10^{-4} \mathrm{Scm}^{-2}$ for the PPy-IC with a thickness of $4.0 \mu \mathrm{m}$, respectively.

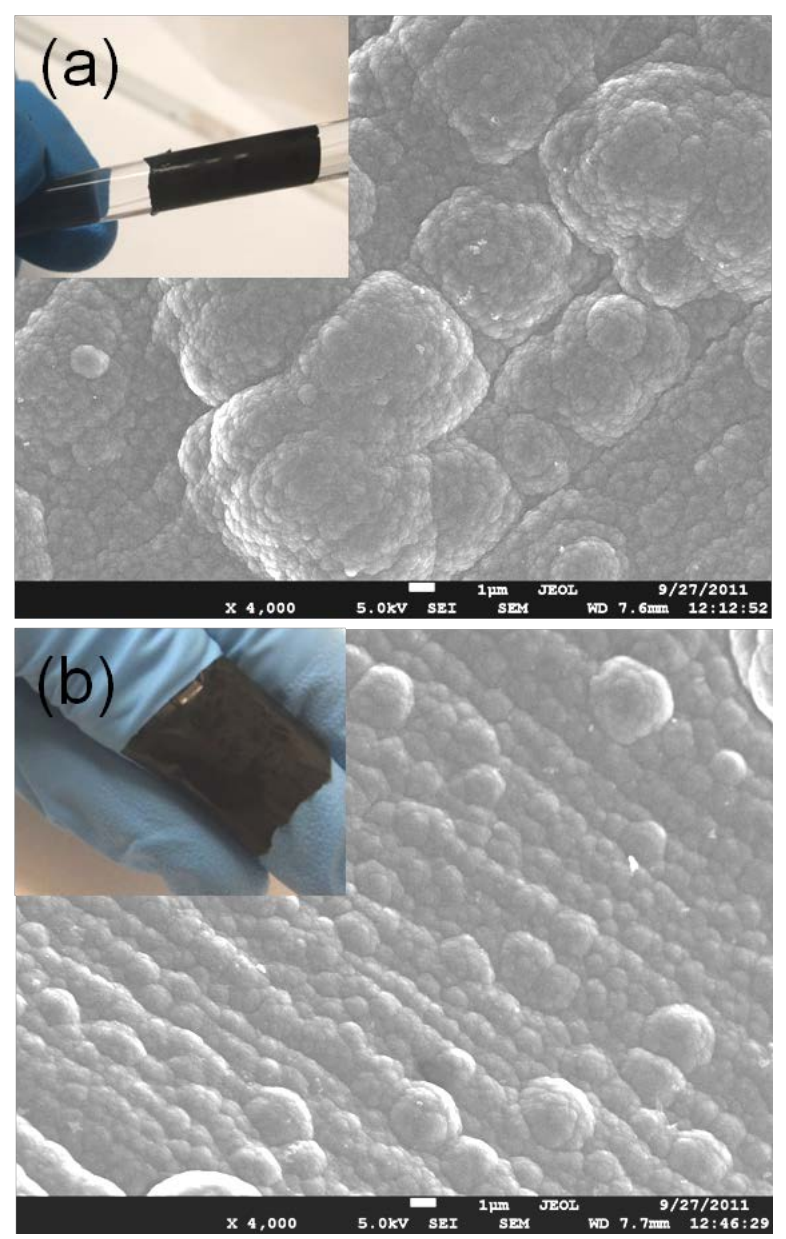

Fig. 3. FESEM images of the films: (a) PPy-pTS film with photograph of rolled-up film (inset) and (b) PPy-IC film with photograph of rolled-up film (inset). 
Polymer batteries using a coin type (CR2032) cells configuration were fabricated and tested. The batteries consist of 1) a PPy-pTS cathode, a PPy-IC anode and an electrolyte of $1 \mathrm{M}$ $\mathrm{LiPF}_{6}$ in an EC-DMC mixture; and 2) a PPy-pTS cathode, a PPy-IC anode and gel polymer electrolyte of PVDF-HFP were tested. The capacity of the polymer batteries was based on the active mass of the anode. The basis of the polymer battery operation is shown in Scheme 1 $[11,14]$. The cathode cycles between the oxidised fully charged conditions of the conducting polymer, in which a charge-compensation anion intercalates to maintain charge neutrality $\left(p \mathrm{TS}^{-}\right)$, and the neutral uncharged condition. The anode cycles between the neutral fully charged condition with a charge compensation intercalated cation $\left(\mathrm{M}^{+}=\mathrm{Li}^{+}\right)$and the oxidised condition. The reaction at the anode and cathode during discharge in a cell with $\mathrm{LiPF}_{6}$ as the electrolyte can be represented as:

Anode:

$\left[\mathrm{PPy}^{0} / \mathrm{IC}^{-}\right] \mathrm{Li}^{+} \rightarrow \mathrm{PPy}^{+} / \mathrm{IC}^{-}+\mathrm{Li}^{+}+\mathrm{e}^{-}$

Cathode:

$\left.\left[\mathrm{PPy}^{+}\right] p \mathrm{Ts}^{-}\right]+\mathrm{e}^{-} \rightarrow\left[\mathrm{PPy}^{0}\right]+p \mathrm{Ts}^{-}$
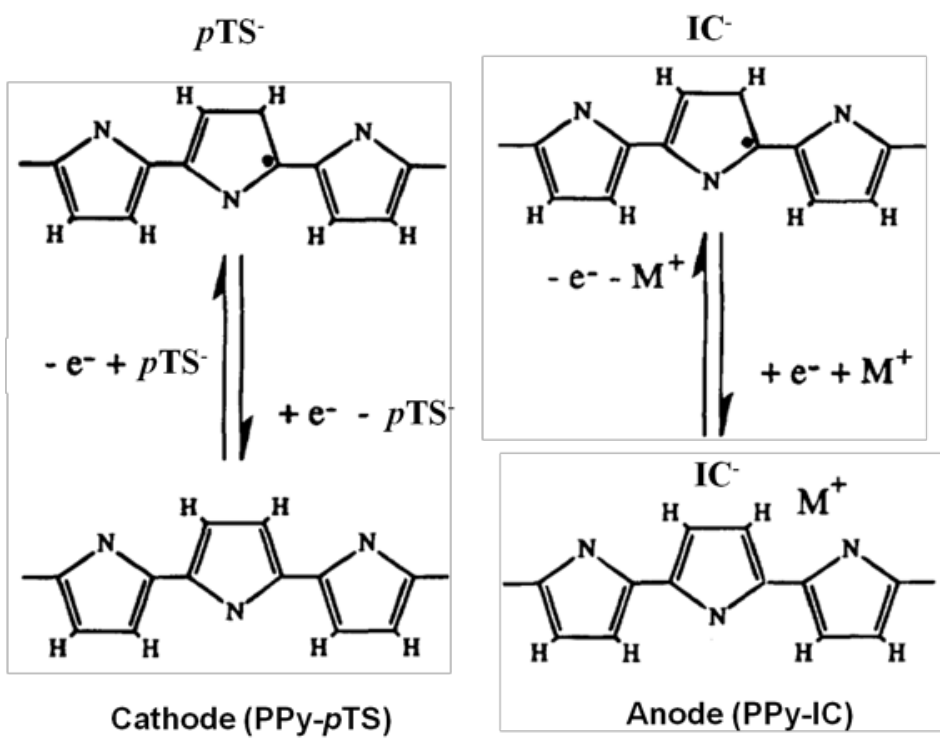

Scheme 1. Oxidation/reduction schemes for the PPy-pTS/PPy-IC system. 
Galvanostatic charging and discharging were used to evaluate the capacity of the system. The cell voltage decreases/increases as the depth of the discharging/charging is increased respectively, which is characteristic of rechargeable batteries. Fig. 4 (a) compares the cycling stabilities of the PPy-pTS/PPy-IC (commercial electrolyte) system at $0.05 \mathrm{~mA} \mathrm{~cm}^{-2}$ with that of the PPy-pTS/PPy-IC (polymer electrolyte) in the voltage range of $0.01-3.0 \mathrm{~V}$, and their corresponding initial discharge-charge voltage profiles are shown in Fig. 4 (b). The PPypTS/PPy-IC (commercial electrolyte) system shows an initial discharge capacity of $39 \mathrm{mAh}$

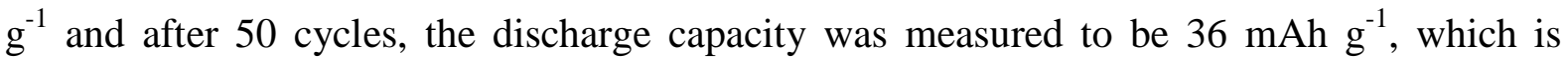
around $92 \%$ of the initial discharge capacity. In the case of PPy-pTS/PPy-IC (polymer electrolyte) system, the discharge capacity retention was $16 \mathrm{mAh} \mathrm{g}^{-1}$, which is also $76 \%$ of the intial discharge capacity. However, the capacity degradation rate was calculated only 0.16 and $0.48 \% / c y c l e$ for the PPy-pTS/PPy-IC (commercial electrolyte) and PPy-pTS/PPy-IC (polymer electrolyte) system, respectively, confirming stable cycling performance of the both systems. The cyclic voltammograms of PPy-pTS/PPy-IC (commercial electrolyte) (Fig. 4 (c)) and PPy-pTS/PPy-IC (polymer electrolyte) (Fig. 4 (b)) systems with a scan rate of $0.01 \mathrm{mV} / \mathrm{s}$ are also presented in this study. The cathodic peaks (reduction peaks) located at around 0.5 and 1.85 V for the PPy-pTS/PPy-IC (commercial electrolyte), and 0.5 and $2.05 \mathrm{~V}$ for the PPy-pTS/PPy-IC (polymer electrolyte) systems correspond to the voltage platform of the discharge process, whereas, the anodic peaks (oxidation peaks) located at around 1.06 and $1.98 \mathrm{~V}$ for the PPy-pTS/PPy-IC (commercial electrolyte), and 1.2 and $2.5 \mathrm{~V}$ for the PPypTS/PPy-IC (polymer electrolyte) systems correspond to the voltage platform of the charge process, respectively. The cathodic/anodic peaks in the cyclic voltammograms are in good agreement with the plateaus observed in the discharge-charge voltage profiles (Fig. 4 (b)). Compared with $\quad$ PPy-pTS/PPy-IC (commercial electrolyte), the major redox peak of Py- 
pTS/PPy-IC (polymer electrolyte) are slightly shifted, which may be due to the effect of polymer electrolyte.
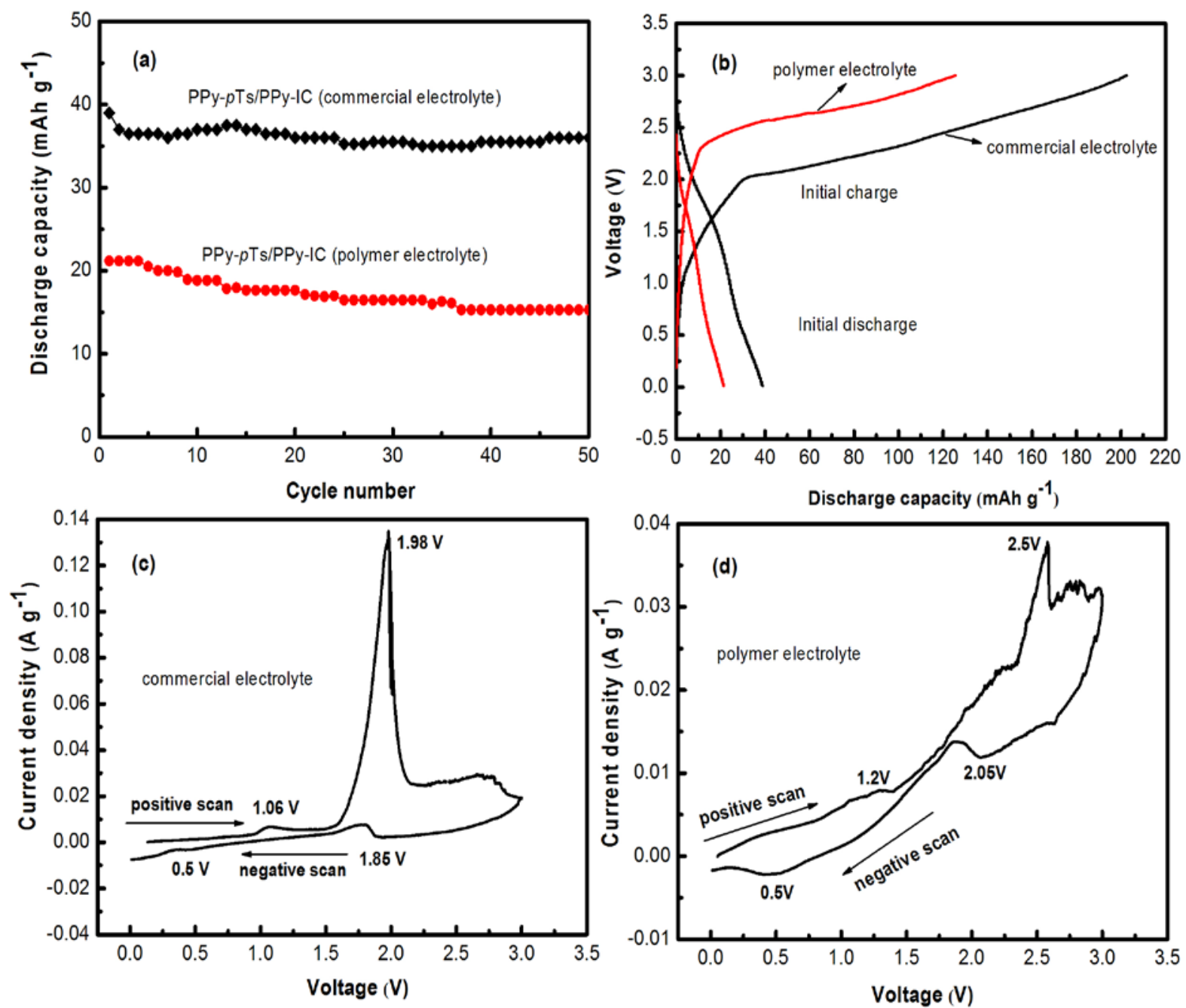

Fig. 4. Electrochemical performances of PPy-pTS/PPy-IC (commercial electrolyte) and PPy-pTS/PPy-IC (commercial electrolyte) system at 0.01-3.0 V: (a) cyclic performance up to 50 cycles at $0.05 \mathrm{~mA} \mathrm{~cm}^{-2}$; (b) initial galvanostatic charge-discharge voltage profiles; (c and d) cyclic voltammograms at a scan rate of $0.01 \mathrm{mV} / \mathrm{s}$.

Electrochemical impedance spectroscopy (EIS) was performed on PPy-pTS/PPy-IC (commercial electrolyte) and PPy-pTS/PPy-IC (polymer electrolyte) systems. The impedance response exhibits a semicircular loop in the medium frequency region. The diameter of this 
semicircle gives the charge-transfer resistance (Rct), which is a measure of the chargetransfer kinetics. We found that the diameter of the semicircle in the medium frequency region for PPy-pTS/PPy-IC (commercial electrolyte) is much smaller than for the PPypTS/PPy-IC (polymer electrolyte) which indicates the lower charge transfer resistance for the PPy-pTS/PPy-IC (commercial electrolyte) system.
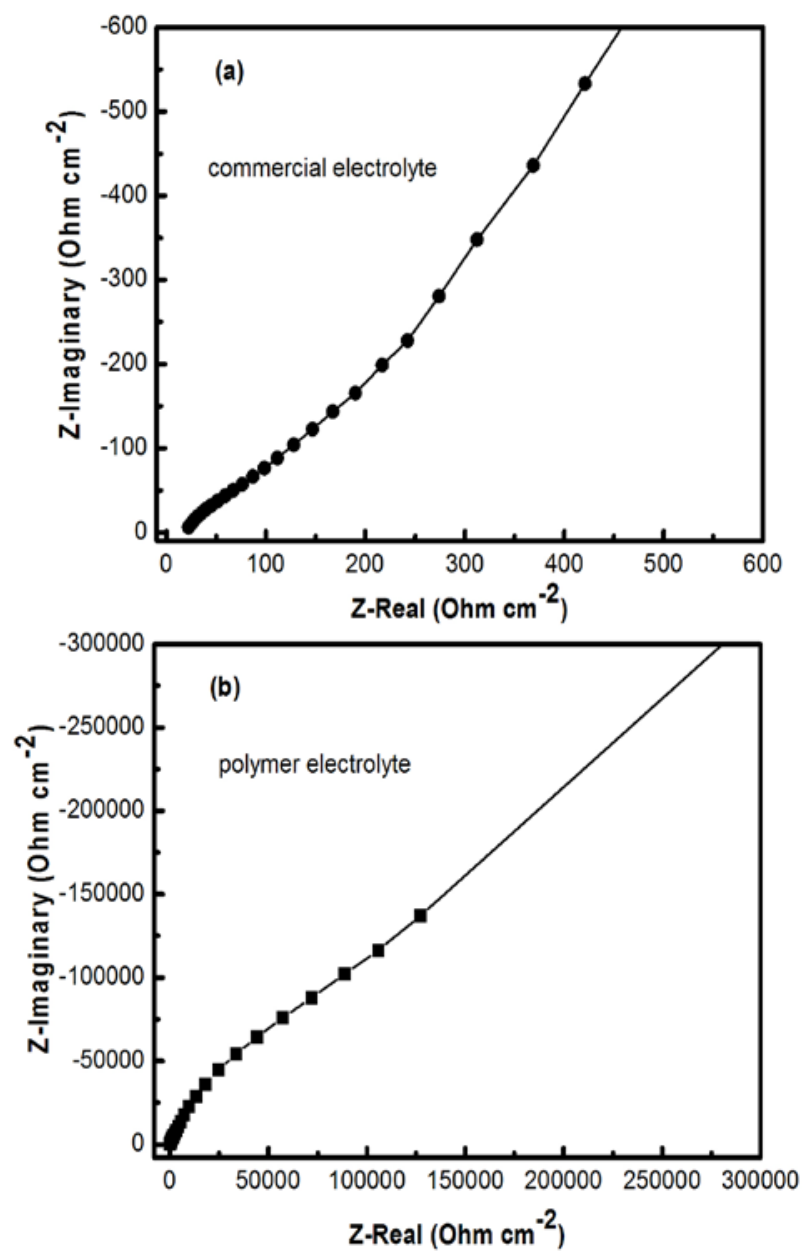

Fig. 5. Impedance plots for the PPy-pTS/PPy-IC (commercial electrolyte) and PPy-pTS/PPyIC (commercial electrolyte) systems.

The good cycling response for the both systems is well supported by the FESEM micrographs of the cycled films of PPy-pTS/PPy-IC (commercial electrolyte) and PPy-pTS/PPy-IC (polymer electrolyte) systems are shown in Fig. 6. From this FESEM observation, it is clearly 
seen that the cathode and anode films for the both systems maintain excellent structural integrity (devoid of any cracks) even after 50 charge-discharge cycles (compares Fig. 6 with Fig.3). Moreover, there appears to be no significant change in a cauliflower-like structure composed of microspherical grains and the size of the nodules after cycling, which indicates no new surfaces generated during cycling. The excellent structural integrity of the films is expected to arise may be due to more homogeneous distribution of anions (dopants) in a molecular level which increase the structural stability and mechanical strength of PPy matrix [19].
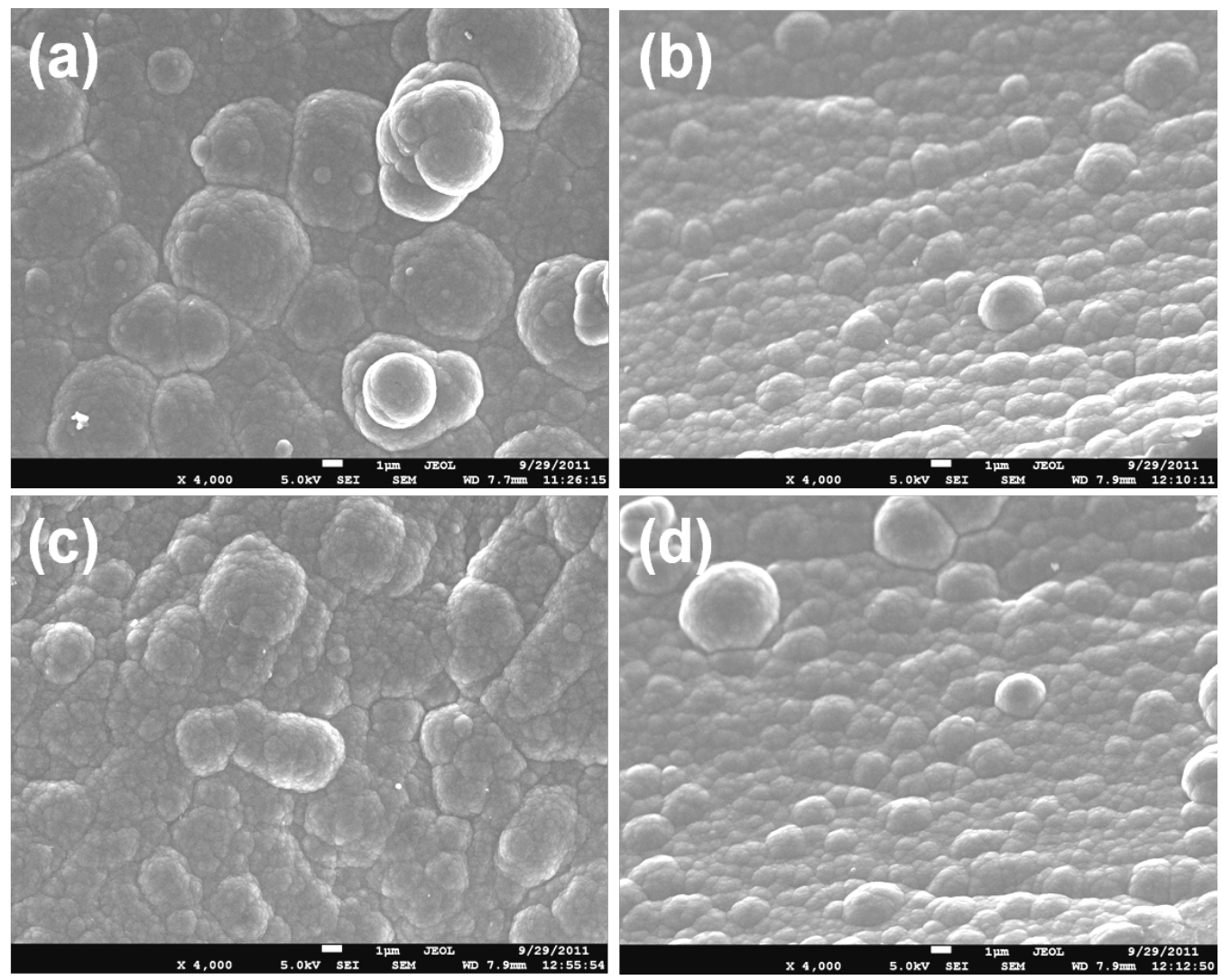

Fig. 6. FESEM images of the electrodes taken after 50 charge/discharge cycles at $0.05 \mathrm{~mA}$ $\mathrm{cm}^{-2}$ : (a, b) images of PPy-pTS/PPy-IC (commercial electrolyte) system, where (a) image of PPy-pTS and (b) image of PPy-IC film electrode respectively; (c, d) images of PPy-pTS/PPy- 
IC (polymer electrolyte) system, where (c) image of PPy-pTS and (d) image of PPy-IC film electrode respectively.

\section{Conclusions}

In conclusion, PPy-pTS and PPy-IC films have been successfully prepared via electropolymerization using the galvanostatic technique. The morphology of the films features a cauliflower-like structure composed of microspherical grains, which could be related to dopant intercalation in the polymeric chain. The films are mechanically robust, lightweight, and flexible. It can be molded into various shapes and its thin sheets can be rolled to make very compact energy storage devices. We believe that the concept presented here, the molecular-level incorporation of electroactive molecules into conducting polymers, can provide a spectrum of materials useful for energy storage devices. The present battery system holds great promise for applications in areas of roll-up displays, medical devices, wearable displays, embedded health monitoring devices and wearable military devices.

\section{Acknowledgements}

The authors are grateful for funding from the Australian Research Council (ARC) under an ARC Centre of Excellence Program (CE0561616). The authors also thank Dr. T. Silver for critical reading of the manuscript.

\section{References}

[1] D. Liu, G. Cao, Energy Environ. Sci. 3 (2010) 1218-1237.

[2] M.M. Rahman, J.Z. Wang, M. F. Hassan, S.L. Chou, Z. Chen, H. K. Liu, Energy Environ. Sci. 4 (2011) 952-957. 
[3] B. Scrosati, J. Garche, J. Power Sources 195 (2010) 2419-2430.

[4] P. Novák, K. Müller, K. S. V. Santhanam, O. Haas, Chem. Rev. 97 (1997) 207-282.

[5] L. Nyholm, G. Nyström, A. Mihranyan, M. Strømme, Adv. Mater. 23 (2011) 37513769.

[6] G. A. Snook, P. Kao, A. S. Best, J. Power Sources 196 (2011) 1-12.

[7] A. Rudge, J. Davey, I. Raistrick, S. Gottesfeld, J. P. Ferraris, J. Power Sources 47 (1994) 89-107.

[8] T. Shimidzu, A. Ohtani, T. Iyoda, K. Honda, J. Chem. Soc Chem. Commun. (1987) 327-328.

[9] C. Zhong, K. Doblhofer, Electrochim. Acta 35 (1990) 1971-1976.

[10] T. Shimidzu, A. Ohtani, P Iyoda, K. Honda, J. Electroanal. Chem. 224 (1987) 123135.

[11] J. G. Killian, B. M. Coffey, F. Gao, T. O. Poehler, P. C. Searson, J. Electrochem. Soc. 143 (3) (1996) 936-942.

[12] C. Y. Wang, G. Tsekouras, P. Wagner, S. Gambhir, C. O. Too, D. Officer, G. G. Wallace, Synth. Met. 160 (2010) 76-82.

[13] C. Y. Wang, A. M. Ballantyne, S. B. Hall, C. O. Too, D. L. Officer, G. G. Wallace, J. Power Sources 156 (2006) 610-614.

[14] J. Wang, C. O. Too, G. G. Wallace, J. Power Sources 150 (2005) 223-228.

[15] J. Z. Wang, S. L. Chou, J. Chen, S. Y. Chew, G. X. Wang, K. Konstantinov, J. Wu, S. X. Dou, H. K. Liu, Electrochem. Commun. 10 (2008) 1781-1784.

[16] J. Z. Wang, S. L. Chou, H. Liu, G. X. Wang, C. Zhong, S. Y. Chew, H. K. Liu, Mater. Lett. 63 (2009) 2352-2354.

[17] H.K. Song, G. T. R. Palmore, Adv. Mater. 18 (2006) 1764-1768. 
[18] A.S. Liu, M.C. Bezerra, L.Y. Cho, Materials Research 12 (4) (2009) 503-507.

[19] V. K. Gade, D. J. Shirale, P. D. Gaikwad, K. P. Kakde, P. A. Savale, H. J. Kharat, M. D. Shirsat, International Journal of Polymeric Materials 56 (2007) 107-114. 